\title{
UNSEEN VISIBLE WATERMARKING IN 2D IMAGES USING RIDGELET TRANSFORM
}

\author{
Sivapriya.C ${ }^{1}$, Vignesh. $\mathbf{R}^{2}$, Tejaswini. ${ }^{3}$, Nithya. $\mathbf{M}^{4}$ \\ ${ }^{1}$ Student, ECE Department, Saveetha School of Engineering, TamilNadu, India \\ ${ }^{2}$ Assistant Professor, ECE Department, Saveetha School of Engineering, TamilNadu, India \\ ${ }^{3}$ Student, ECE Department, Saveetha School of Engineering, TamilNadu, India \\ ${ }^{4}$ Student, ECE Department, Saveetha School of Engineering, TamilNadu, India
}

\begin{abstract}
The Watermarking is the method of embedding data into digital multimedia content. It is used to verify and recognize the identity of the digital content's owner. Real time watermarks that are embedded into physical objects like currencies or bills or letter papers are invisible under normal viewing conditions. But if the viewing condition changes, watermark patterns will become visible. Some watermarks can be viewed only if the saturation level of that image is increased. Such type is known as unseen visible watermarking $(U V W)$. In this watermarking method, the $2 D$ images are watermarked by UVW method using Ridgelet wavelet transform to increase the efficiency. The quality of Watermarking is determined by important factors namely robustness, capacity, imperceptibility and blindness.
\end{abstract}

Keywords: Watermarking, Ridgelet, Imperceptibility

\section{INTRODUCTION}

The progress in graphics media and storage technology has allowed the storing and transmitting of an increasing amount of information in the digital format. However the ease of copy and reproducing digital data is likely to encourage violation of Copyright.[3] The purpose of watermark in digital image is to provide security and rightful ownership for intellectual property that is in the digital format. ${ }_{[4]}$ Moreover, only human-readable information can be conveyed by existing visible watermarking schemes, since automatically distinguishing faint watermark patterns from marked visual contents would be a very difficult task. The main factors used to determine the quality of watermarking are robustness, imperceptibility, capacity and blindness. This work deals with the unseen invisible watermarking of $2 \mathrm{D}$ images. It is done using MatlabSimulink . The blueprint of the paper is as follows: Section II tells about the Wavelets. Section III presents about the methods used. Ridgelet transform is explained in section IV. Block diagram is explained in section V. Algorithm used is explained in section VI. Output display in section VII and the parameters calculated are mentioned in section VIII. The result is presented in section IX and at last the paper is concluded in section $\mathrm{X}$.

\section{WAVELET TRANSFORM}

A wavelet is a function helpful in digital signal processing and image processing. The wavelet transform is used to convert the image in time-frequency representation. The use of wavelets has now reached great heights. The principles used are similar to those of Fourier analysis. Unlike Fourier transform, in wavelets, a single function is used and its dilations and translations are used to generate a set of orthonormal functions to represent a signal. Various types of wavelets are used for signal analysis. Choice of a particular wavelet depends upon the type of application. Wavelets are widely used in signal processing, data compression, speech recognition. [5]

The different types of wavelets are Haar, Shannon, Meyer, Daubechies, Coiflets, Biorthogonal, Mexican Hat, Symlet and Ridgelet wavelet.[6]

\section{METHODS USED}

\section{A.Existing Method}

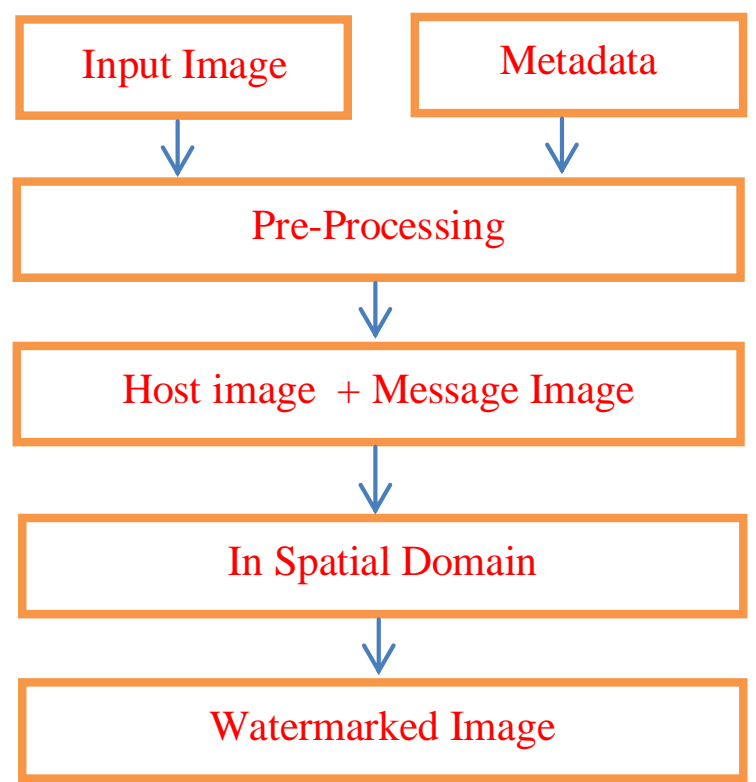

Figure 1: Existing method 


\section{B.Proposed Method}

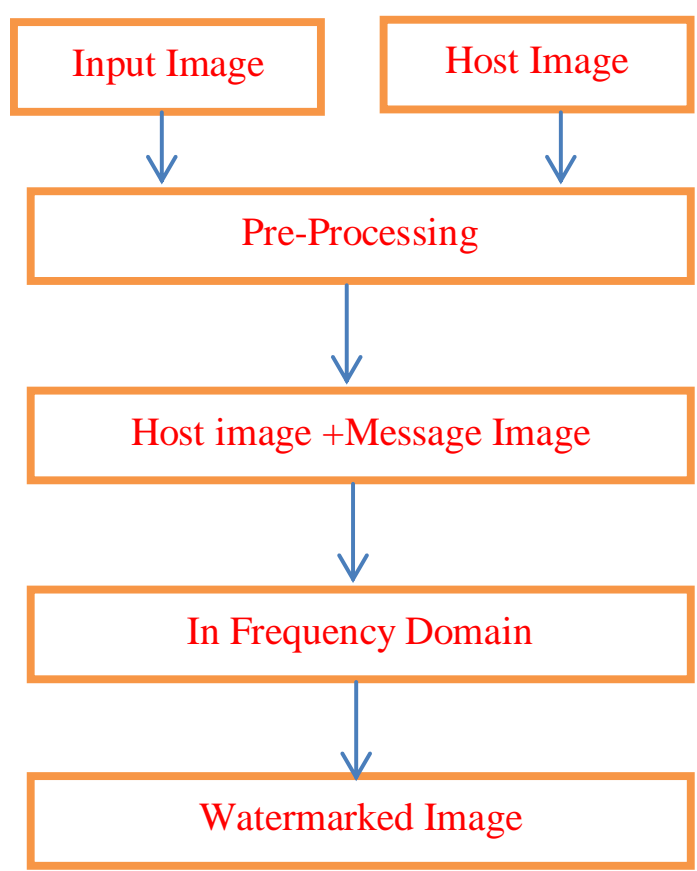

Figure 2: Proposed method

In the proposed method, frequency domain is preferred over spatial domain. The main aim is to embed the watermark in the spectral coeffecients of the message image

\section{NEED OF RIDGELET TRANSFORM}

The Ridgelet wavelet is a type of wavelet defining a discrete transform of wavelets and characterized by a maximum amount of vanishing moments for some given support.

The idea is to mark a line into a point singularity using the Radon transform . Then, the wavelet transform can be used to handle the point singularity.

The ridgelet transform is the application of 1-D wavelet transform to the parts of the Radon transform, whereas the 2-D wavelet transform is the application of 1-D transform and the Radon slices.[1]

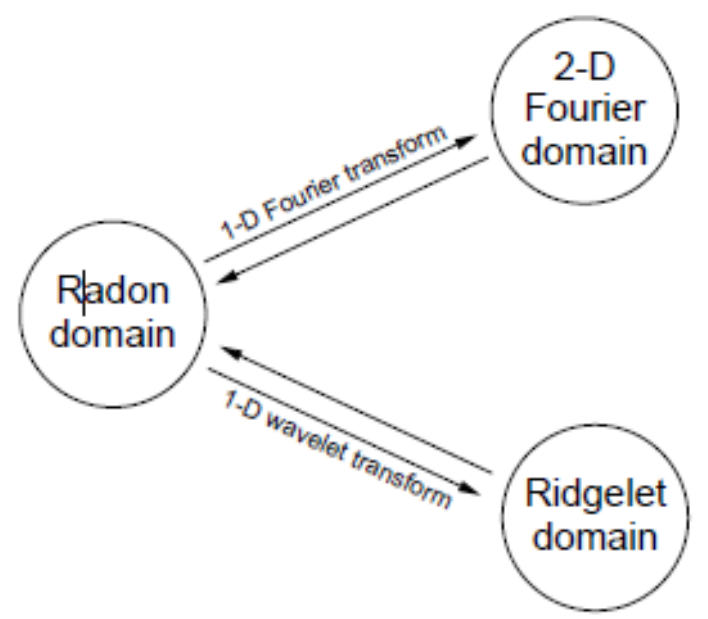

Figure 3: Ridgelet transform
Because of their ability to better represent signals, Ridgelet transforms are preferred than any other wavelet transforms in general. Ridgelet transform enhances the idea of point-topoint to point-to-line mappings of singularities, which is more effective in handling.

To obtain a balance between imperceptibility and robustness, most methods embed watermark in ridgelet transform. [2] Hence the two dimensional images are watermarked using Ridgelet wavelets.

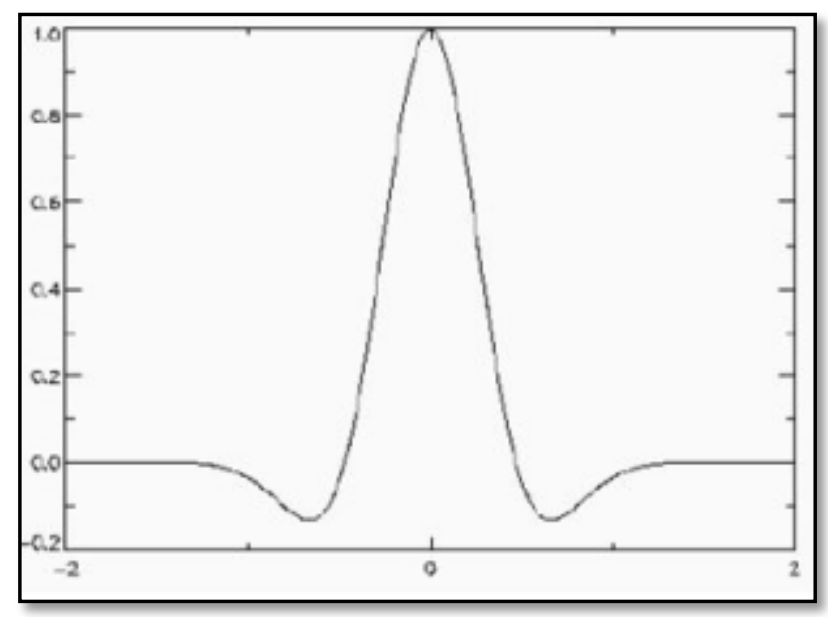

Fig 4: Characteristic curve of RidgeletWaveletThe Continuous Ridgelet Transform (CRT) in R2 is defined as

$\mathrm{CRT}_{\mathrm{f}}(\mathrm{a}, \mathrm{b}, \theta)=\int \Psi(x) f(x) d x$

where the ridgelets $\Psi a, b, \theta$ (x) in $2 \mathrm{D}$ are defined from a wavelet-type function in $1 \mathrm{D}, \psi(\mathrm{x})$, as

$\Psi \mathrm{a}, \mathrm{b}, \theta(\mathrm{x})=\frac{1}{(\sqrt{a})} \Psi \frac{(\mathrm{x} 1 \cos \theta+\mathrm{x} 2 \sin \theta-b)}{a}$

where $x=(x 1, x 2)^{T}$. In Equations (1) and (2), the parameters $\mathrm{a}, \mathrm{b}$ and $\theta$ relate to scaling, shift and rotation transforms respectively.[7]

\section{BLOCK DIAGRAM}

The following figure is the block diagram of Unseen visible watermarking(UVW) .Each block is explained below. 

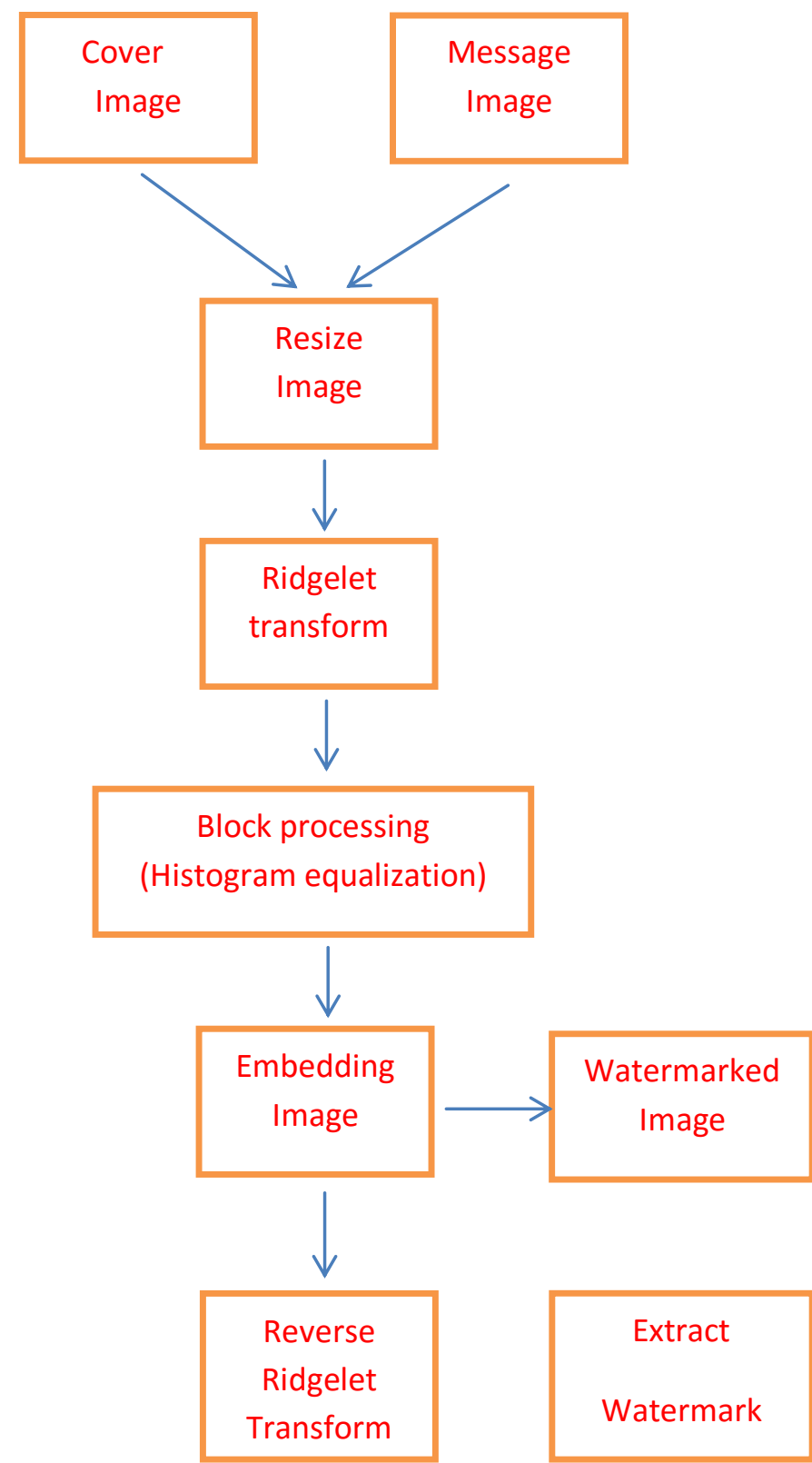

Fig 5: Block diagram of UVW

\section{A. Cover image}

The cover image is the original image that is to be watermarked. The message image is the image to be hidden as a watermark.

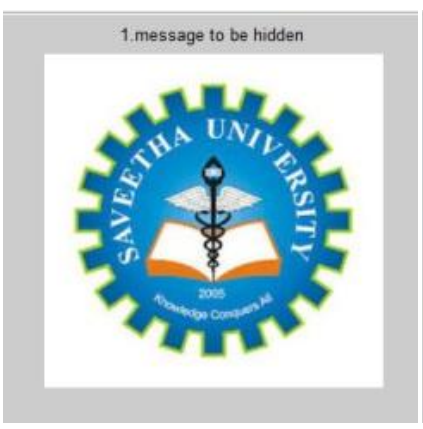

(a)Message

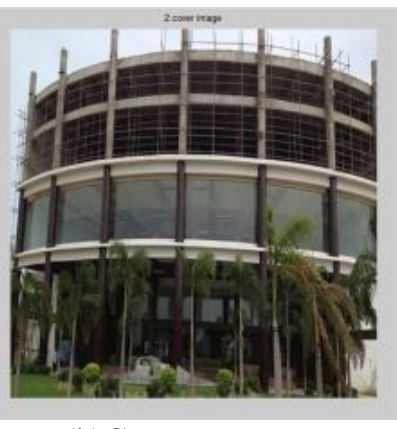

(b)Cover
Fig 6: Message image and cover image

\section{B.Image Resize}

The cover image and the message image may be of various sizes. Hence the message and cover image has to be resized to perform the watermarking .

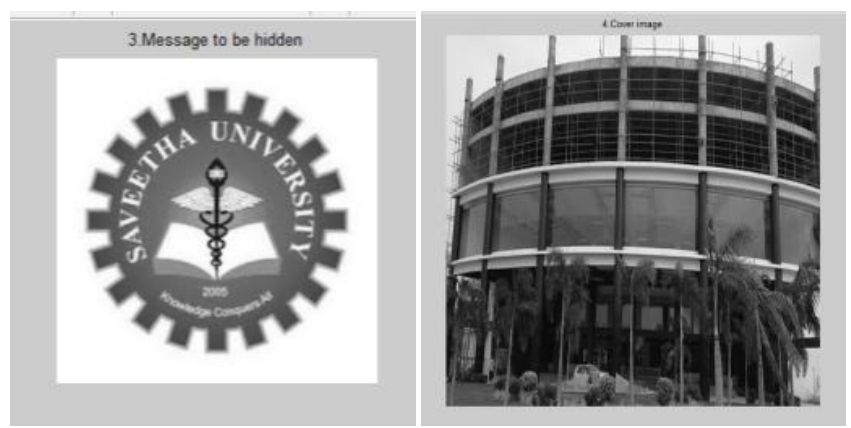

Fig 7: Resized image

\section{C.Ridgelet transform}

Ridgelet transform is applied on the cover image to compact the image into a small number of pixels. During the ridgelettransform ,the image is processed and multiplied with the alpha factor.

Hence the values will be in the form of decimal and exponential thus the images are converted into double.
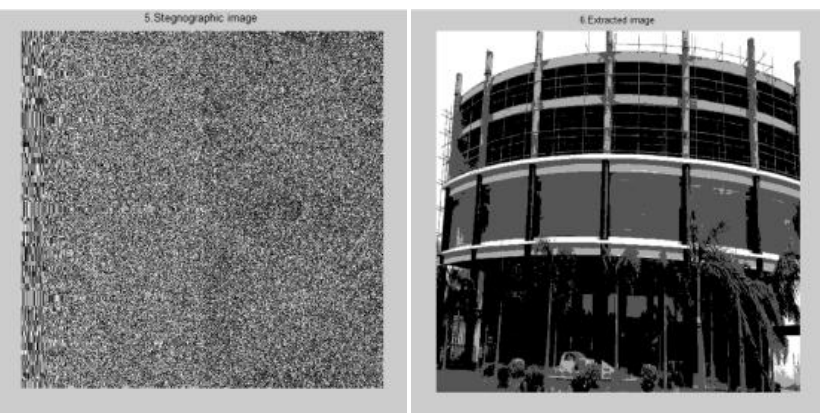

Fig 8: Transformed image

\section{Block processing}

In this stage, the images are converted into gray image and histogram equalization is done. A histogram is a graphical representation of the distribution of numerical data. Histogram equalization is one of the method used in image processing for the brightness adjustment using the image's histogram. This method usually increases the contrast of images, mainly when the data of the image is represented by close contrast values. By this adjustment, the intensities can be better distributed on the histogram.

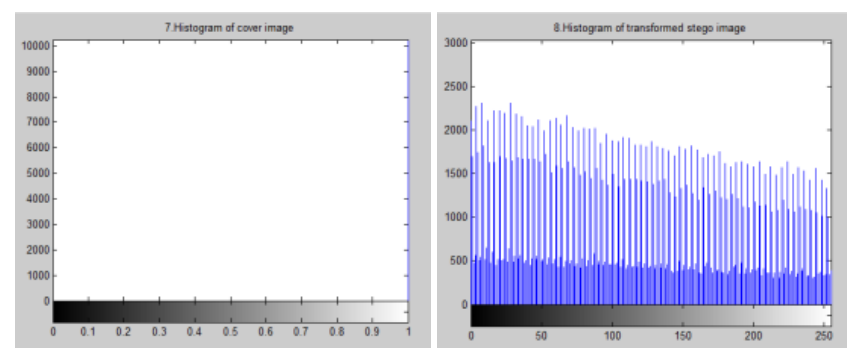

Fig 9: Processed image 


\section{E.Embedding Image}

Embedding the message image in the cover image is the fourth step of the watermarking process. The message image is embedded in the cover image bit by bit and then stored.

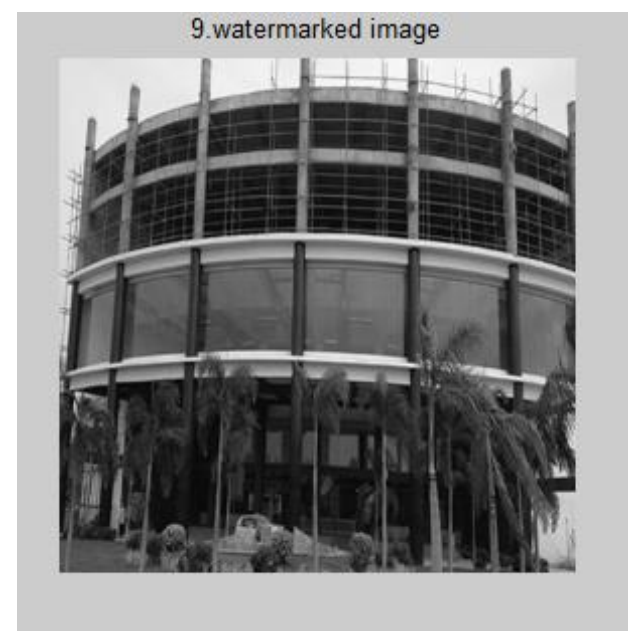

Fig 10: Embedded image

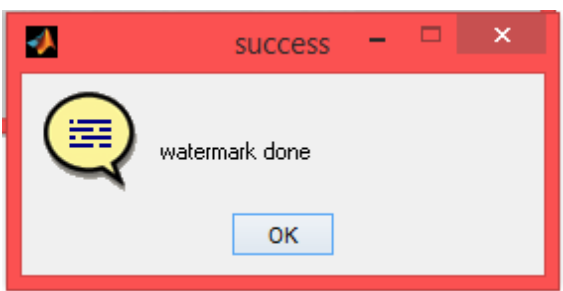

Fig 11: Output display

\section{F.Extract image}

Watermark extraction is the process of extracting the original image from the watermarked image. Reverse ridgelet transform is used to extract the original image from the watermarked image. During extraction, the bits are shifted in the reverse order to get the original image.

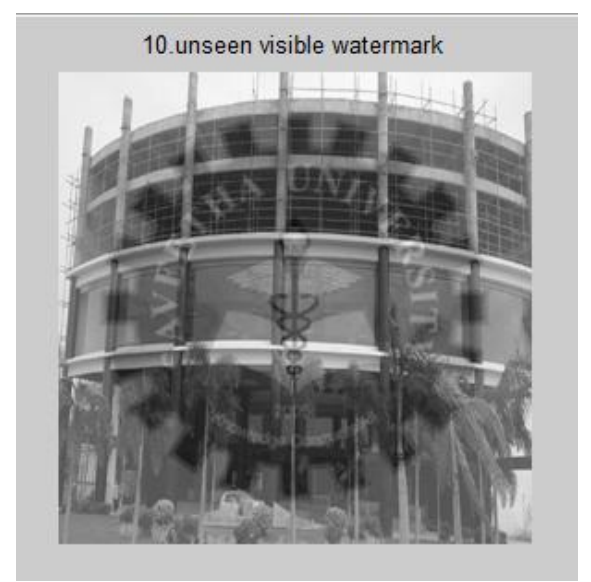

Fig 12: Visible watermark

\section{ALGORITHM USED}

The algorithm used here is the least significant bit, shortly called as LSB algorithm. It is used along with ridgelet transform to watermark the images. In this type, the watermark is embedded bit by bit in the cover image.

\section{OUTPUT DISPLAY}

Thus in the unseen visible watermarking, the original image and the watermarked image looks similar. Hence the watermark can be viewed only if the brightness of the image is increased.

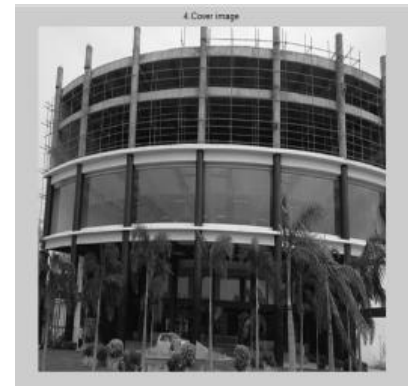

(a) Unseen watermark

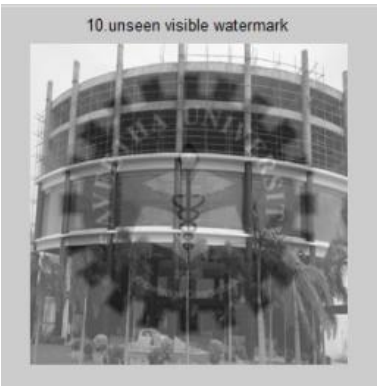

(b) Visible watermark
Fig 13: Watermarked image

Depending upon the values of the alpha factor, the contrast of the image is varied in accordance with the watermarked image.

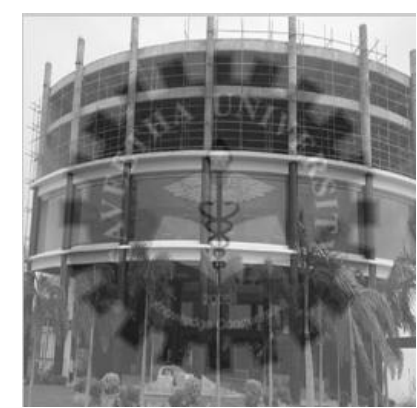

Fig 14: Output with threshold $=45$ and alpha $=0.3$

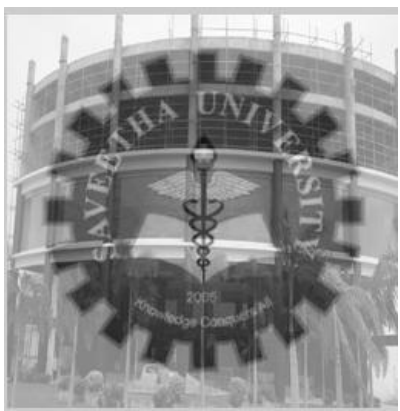

Fig 15: Output at threshold $=45$ and alpha $=0.3$

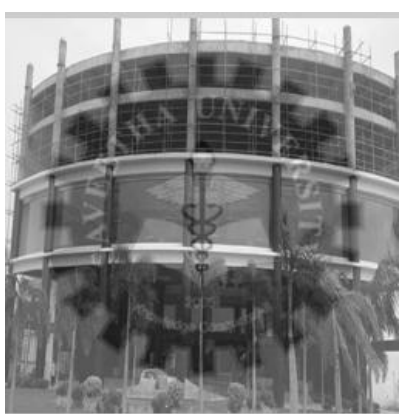

Fig 16: Output at threshold $=65$ and alpha $=0.9$ 


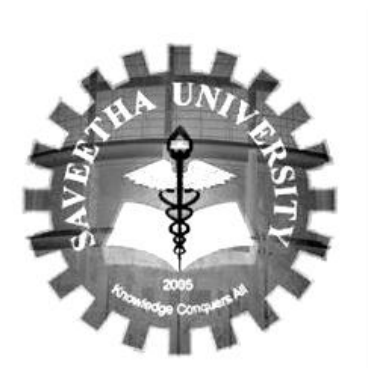

Fig 17: Output at threshold $=95$ and alpha $=1.5$

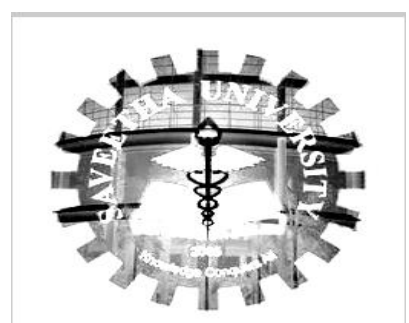

Fig 18: Output at threshold $=55$ and alpha $=2.5$

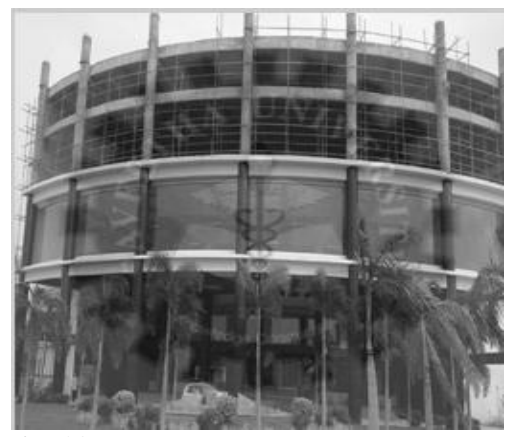

Fig 19: Output at threshold $=55$ and alpha $=0.2$

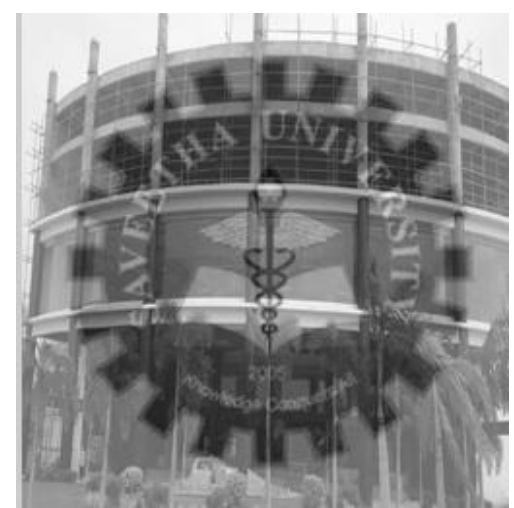

Fig 20: Output at threshold $=65$ and alpha $=0.3$

\section{PARAMETERS CALCULATED}

The performance analysis of the watermarking is done based on the parameters like Peak signal to noise ratio (PSNR) and Mean square error (MSE), Robustness and perceptuality. Robustness means the measure of resistance of watermarked image against attacks to destroy the watermarked image and modification like filtering, scaling.
Perceptuality means the quality of the image should not be destroyed the presence of watermark. The quality of the watermark is calculates by using PSNR. Higher the PSNR, better the quality of the watermarked image. The PSNR of the image can be calculated using the formula below

$\operatorname{PSNR}=\frac{10 * \log 10\left(\text { peak }^{2}\right)}{M S E}$

where MSE is the average of the squared errors between actual and estimated readings in the given data.

The PSNR of the extracted image is 23.0195 and the Mean Square Error (MSE) of the image is $1.3207 \mathrm{e}+03$

\section{RESULT}

The unseen visible watermarking is done using Ridgelet wavelet transform and the parameter like Peak Signal to Noise Ratio (PSNR), Mean Square Error (MSE) are calculated.

\section{CONCLUSION}

Ridgelet wavelet watermark method provides better result than wavelet for watermarking in digital images. In case of multimedia applications, watermarking using wavelets are achieved with low resolution. This project can also be enhanced by using Haar wavelet and Shannon wavelet in future.

\section{REFERENCES}

[1] Thaarini.P, and Thiyagarajan.J. "Comparative Analysis of Image Compression Using Wavelet and Ridgelet Transform" Second National Conference on Trends in Automotive Parts Systems and Application,2014.

[2] EsaRahtuJanneHeikkila, MikkoSalo ," A New Affine Invariant Image Transform Based on Ridgelets"ed., vol. 3, J. Peters, Ed. New York: McGraw-Hill,1964,pp.1564.

[3] Chun-Hsiang Huang, Shang-Chih Chuang, Yen-Lin Huang, and Ja-Ling Wu,,'Unseen Visible Watermarking: A Novel Methodology for Auxiliary Information Delivery via Visual Contents", IEEE

[4] Yu-Hsun Lin ,Ja-Ling Wu,"Unseen visible watermarking for color plus depth 3D images", Graduate Institute of Networking and Multimedia, National Taiwan University

[5] ChethanParameswariah, and Mickey Cox,"Frequency characteristics of wavelets",IEEE

[6] Minh N.Do and Martin Vetterli," The Finite Ridgelet Transform for Image Representation", member, IEEE

[7] PooyaMonshizadehNaini,"Digital watermarking using MATLAB", University of Thran, Iran.

[8] SatyajitKautkar, RahulkumarKoche, TusharKeskar, AniketPande, MilindRane, Gary A. Atkinson' "Face recognition based on Ridgelet transform", ${ }^{\text {Department }}$ of Electronics, VIT, Pune 